\title{
Application of Geographical Information System and Remote Sensing in Veterinary Parasitology
}

\author{
S.R. Tramboo ${ }^{1}$, Z.A. Wani*2, R.A. Shahardar ${ }^{3}$, I.M. Allaie $^{2}$ and K.H. \\ Bulbul $^{2}$
}

Division of Veterinary Parasitology, Sher-e-Kashmir University of Agricultural Sciences and Technology of Kashmir, Shuhama Campus, Jammu and Kashmir 190 006, India,

$*^{2}$ Corresponding author e-mail: zahoorwani_103@yahoo.co.in)

Journal of Livestock Science (ISSN online 2277-6214) 13: 12-19

Received on 29/7/21; Accepted on 16/12/21; Published on 8/1/22

doi. 10.33259/JLivestSci.2022.12-19

\begin{abstract}
The satellite remote sensing being a newly technology has been developed in view of serious pressures on our natural habitat. This terminology was used as early as 1960s has been defined as the sum total of processes used to acquire and measure the information of some property of objects and phenomena by a recording device (sensor) which is not in physical contact with the objects and phenomena in study. Basically, the process is a combination of an object surface, the recording device and the information carrying energy waves. For the last couple of decades, the application of remote sensing has not only revolutionized the way data have been collected, but also significantly improved the quality and accessibility of information for conservation and management of natural resources. The parallel advance in the reliability of GIS has permitted the interpretation of large quantity of data generated through remote sensing to address different environmental problems particularly mapping of disease incidence, prevalence, and mortality and morbidity etc. thus remote sensing could be used to check the spread of diseases and then application of immediate control measures. A very useful function of GIS which helps in modeling the diseases is the kriging. Kriging has been widely used in Veterinary Parasitology to model the distribution of various parasites/ diseases, e.g. Canine Heartworm infection in Europe, Ixodes scapularis, malaria, alveolar echinococcosis, tsetse flies, Calicophoron daubneyi and Oncomelania hupensins. Although remote sensing and GIS of bio invasion mapping has lead to the development of a diverse range of mapping technique, but in terms of real world applications, the technology needs further development.
\end{abstract}

Key words: Geographical Information; Remote sensing; Disease mapping; Parasitology 


\section{Introduction}

Remote sensing is the science as well as art of acquiring earth's surface information without actually being in contact with it. This involves sensing and recording reflected or emitted energy and processing, analyzing and applying that information (Kumar, 2015). A well defined system designed to capture, store, manipulate, analyze, manage and present all types of spatial or geographical data is collectively known as geographic information system (GIS). The term is also used for geographical information science or geospatial information studies, which is a large domain within the discipline of Geo-informatics (Kenneth and Margaret, 2015). It can also be defined as a computer based system that allows us to map, model, query, and analyze large quantities of data within a single database according to their location. Creating maps, integrating information, visualizing scenarios, presenting powerful ideas, and developing effective solutions are the abilities of GIS and its applications are related various fields like engineering, planning management, transport, insurance, telecommunications, and business (Maliene et al., 2011). The International Society for Photogrammetry and Remote Sensing (ISPRS) is an international nongovernmental organization that enhances international cooperation between the worldwide organizations with interests in the photogrammetry, sensing and spatial information sciences. The Indian Institute of Remote Sensing (IIRS) which is an important constituent unit of Indian Space Research Organisation (ISRO) was established in 1966 and plays a key role for training and capacity building in geospatial technology and its applications through training, education and research in Southeast Asia. ISRO maintains one of the biggest fleets of remote sensing (IRS) and communication (INSAT) satellites catering to the needs of the nation through a network of centres, offices, and research institutes in different parts of the country. ISRO functions in the areas like broadcasting, weather forecasting, disaster management, geographic information systems, navigation, cartography (maps), telemedicine, distance education satellites, etc.

In modern era, the term refers to the use of aerial sensor technologies for detecting and classifying objects on Earth (surface as well as atmosphere and oceans) by means of propagated signals. It can be classified into active remote sensing (when a signal is first emitted from aircraft or satellites (Guo et al., 2013) or passive (eg. sunlight) according to the source of signal they utilize to explore the object (Liu \& Mason, 2009). Passive sensors depend on natural radiations emitted or reflected by the object or surrounding areas. Common source of radiation for passive sensors is reflected sunlight. Film photography, infrared, charge-coupled devices, and radiometers are examples of passive sensors. Active sensors release energy to scan objects and areas and then the sensor detects and measures the radiation that is reflected or backscattered from the target. Examples of active sensors include RADAR, LiDAR, laser altimeter and scatterometer are examples of active remote sensing in which the time delay between emission and return measured establishes the location, speed and direction of an object.

As per Melesse et al. (2007) eight distinct eras of remote sensing which are running parallel in time periods, but are distinctly unique in terms of technology, concept of utilization of data, applications and data characteristics are:

1. Airborne remote sensing era: This era evolved during the First and the Second World War during which remote sensing was mainly used for the purposes of surveying, reconnaissance, mapping and military surveillance.

2. Rudimentary space-borne satellite remote sensing era: It began with the launch of 'test of concept' rudimentary satellites such as Sputnik 1 from Russia and Explorer 1 by the US at the end of 1950s. This was soon followed by the first meteorological satellite called Television and Infrared Observational Satellite-1 (TIROS-1) by the US.

3. Spy satellite remote sensing era: During the peak of the cold war, spy satellites such as Corona were widely used. Its purpose was to collect data almost exclusively for military services. The data were produced as hard copies.

4. The Meteorological satellite sensor remote sensing era: At this time, the early meteorological satellite sensors consisted of geo-synchronous Geostationary Operational Environmental Satellite (GOES) and polar orbiting National Oceanic and Atmospheric 15 Administration (NOAA) and Advanced Very High Resolution Radiometer (AVHRR) were emerged. This was an era when data were developed in digital format and were analyzed using exclusive computer software. This was also an era when global coverage and environmental applications became realistic and practical.

5. Landsat era: The Landsat era began with the launch of Landsat-1 (called Earth Resources Technology Satellite) in 1972 carrying multi-spectral scanner (MSS) sensor. This was followed by other path-finding Landsat satellite 2 and Landsat 3 which carried MSS, and Landsat 4 and Landsat 5, which carried Sensors 2007, 73211 Thematic Mapper (TM). The Landsat 7 carried Enhanced Thematic Mapper (ETM+) sensor, but it failed during the launch. The Landsat-8 carrying Operational Land Imager (OLI) planned for launch in 2011 had equally good sun-synchronous Land satellites such as Systeme Pour l' Observation de Ia Terre (France) and Indian Remote Sensing Satellite (India). These satellites were having high resolution $(2.580 \mathrm{~m})$ and global coverage potential. At this resolution, only 
Landsat is currently gathering data with pole to pole global coverage. This is, by far, the most significant era that kick-started truly wide environmental application of remote sensing data both locally and globally (Janssen and Bakker, 2001).

6. Earth Observing System (EOS) era: This era began with the launch of Terra satellite in 1999 and has brought in the global coverage, frequent repeat coverage, high level of processing, as well as easy and free access to the data. The Terra/Aqua-satellites 16 was carrying sensors such as Moderate Resolution Imaging Spectro-radiometer (MODIS) and Measurements of Pollution in the Troposphere (MOPITT). The active space-borne remote sensing sensors using radar technology include European Radar Satellite (ERS), Japanese Earth Resources Satellite (JERS), Radarsat and Advanced Land Observation Satellite (ALOS).

7. New Millennium era: This era refers to highly advanced 'test-of concept' satellites sent into orbit around the same time as EOS era. These include satellites and sensors of the next generation like Earth Observing- 1 carrying the first space borne hyper spectral data. The idea of Advanced Land Imager (ALI) is technologically a better replacement for Landsat.

8. Private industry era: This has begun at the end of the last millennium and beginning of this millennium and consists of a number of innovations including:

i. Collection of data with very high resolution ( $<10$ meter). This is exemplified by IKONOS and Quick bird satellites.

ii. A revolutionary means of data collection. This is represented by Rapid eye satellite constellation of 5 satellites, having almost daily coverage of any spot on earth at 6.5 meter resolution in 5 spectral bands including a red-edge band.

iii. The introduction of micro-satellites, some under disaster monitoring constellation (DMC), which are designed and launched by Surrey Satellite Technology Ltd. for Turkey, Nigeria, China, US and UK.

iv. The innovation by Google Earth (http://earth.google.com) in making rapid data access of very high resolution image for any part of the world through streaming technology that makes it easily accessible to even non-specialists.

\section{Principle of Remote Sensing}

Detection and discrimination of objects or surface features means detecting and recording radient energy reflected or emitted by objects or surface material. Different objects return different amount of energy in different bands of electromagnetic spectrum incident upon it and it depends on the property of the material (structural, chemical and physical), its surface roughness, angle of incidence, intensity and wave length of radiant energy. The remote sensing is basically a multidisciplinary science which includes a combination of various disciplines such as optics, spectroscopy, photography, computer and electronics and telecommunication satellite launching, etc. All these components are integrated to act as one complete system known as Remote Sensing System. It comprises of a number of stages and each of them is important for its successful operation. The process involves an interaction between incident radiation and targets of interest (Kumar, 2015).

Energy source of illumination: Energy source which illuminates or provides electromagnetic energy to the target of interest is the first requirement for remote sensing.

Radiation and the atmosphere: The energy while travelling from its source to the target will come in contact with the atmosphere as it passes through and the interaction occurs $2^{\text {nd }}$ time while energy travels from the target to the sensor.

Interaction with the target: While making its way to the target, the energy travels through the atmosphere, which then interacts with the target depending on the properties of both target and the radiation.

Recording of energy by the sensor: After the energy has been radiated from the target, it then requires a sensor (remote- not in contact with the target) to collect and record the electromagnetic radiation.

Transmission, reception and processing: The energy received by the sensor must be transmitted in electronic form to the receiving and processing station in order to process the data into an image (hard copy and/ or digital).

Interpretation and analysis: These images are interpreted visually and/ or digitally or electronically to extract information about the illuminated target.

Application: The first element of remote Sensing process is achieved when the supplied information has been extracted from the imagery about the target so as to better understand it for revealing the new information. These 7 processes comprise the Remote Sensing Process from beginning to the end. 


\section{GIS use in Veterinary Surveillance}

GIS can be used to produce maps of disease incidence, prevalence, mortality and morbidity on farm, region, or national levels for recording and reporting diseases. In case of an infectious disease outbreak, GIS can provide an excellent tool for location identification of a case as well as all farms at risk within a specified area of the outbreak so that buffer zones can be drawn around those farms within a short time. These zones can also be generated around other risk areas like the roads where infected animals have been driven or around market places. For field veterinarians, the maps can assist in planning the work in current situation, and also for the veterinary authorities how to handle a potential outbreak (Madelaine, 2001). In veterinary sciences, disease mapping, ecological analysis and epidemiological surveillance processing, analyzing and visualizing spatial data are nowadays carried out by GIS and RS tools. Some of the analyzing tools routinely used in GIS for spatial epidemiology include (i) neighborhood analysis (ii) buffer generation (iii) overlay analysis (iv) network analysis(v) surface area and distance calculations and (vi) three-dimensional surface modeling (Ward and Carpenter, 2000).

Remote Sensing provides a unique source of data that can be exploited to characterize climate and land surface variables at different spatial resolutions. It helps in the calculation of vegetation indices, land surface temperatures, atmospheric and soil moisture and rainfall indices, etc. Since the disease vectors are sensitive to changes in these factors as they have specific requirements, so RS can be used to determine their present and future and also predict their distribution. The environmental and climatic features most commonly used for ecological analysis in veterinary epidemiology include NDVI, land cover and land use, elevation, slope, aspect, presence of lakes, rivers and other water bodies, temperatures, rainfall and humidity.

A very useful function of GIS that is helpful in modeling the diseases is the kriging (Berke, 2004). Kriging has been widely used in meteorology to determine values of climate data from observing stations and has also been used in veterinary epidemiology to model the distribution of various parasites/ diseases, e.g. Canine Heartworm infection in Europe (Genchi et al., 2005), Ixodes scapularis that transmits Lyme disease (Nicholson and Mather, 1996), malaria (Kleinschmidt et al., 2000), alveolar echinococcosis (Pleydell et al., 2004), tsetse flies (Sciarretta et al., 2005), Calicophoron daubneyi, (Biggeri et al., 2004), Oncomelania hupensins (Zhang et al., 2005), as well as co-infection of school children in Côte d'Ivoire with $S$.mansoni and hookworm (Raso et al., 2006).

\section{Applications of GIS and RS in Parasitology}

The rate of $D$. immitis maturation to infective third-stage larvae in the mosquito vector(s) depends mainly on the environmental temperature, and there is a threshold of approximately $14^{\circ} \mathrm{C}$, below which development will not proceed (Fortin and Slocombe, 1981). The total environmental heat required for development is expressed in terms of degree-days in excess of this threshold (heartworm development units-HDUs) (Fortin and Slocombe, 1981; Slocombe et al., 1989).The seasonal Heart Worm transmission model formulated by Slocombe et al., (1989) in Canada and reevaluated by Lok and Knight (1998) for larvae to reach infectivity and a maximum life expectancy for a vector mosquito in the United States requires about 130 HDUs and 30 days respectively. From the National Climatic Data Center, temperature records for the period 1977 to 1991 were analyzed by Fortin and Slocombe model (1981) modified by Lok and Knight (1998) from all parts of the Europe. At every station, by using maximum and minimum daily temperatures, mean daily temperature was calculated. A gap of three days was assumed a threshold for calculating HDUs as the data was not always continuously recorded from all the stations. If the threshold value of 130 cumulative HDUs reached in 30 consecutive days only then it was accepted.

The program searched for the decade of the threshold value. The annual average frequency of each decade was calculated, and the number of generations in each station was estimated to identify the hot spot zones of Heart Worm transmission. In addition, the most favorable earliest and latest decades for transmission were selected to identify the best Heart Worm chemoprophylaxis timing. All data layers were spatially interpolated using a linear Kriging model to produce thematic maps. The results indicated that, GIS was able to show focal point of transmission period and temporal dynamics of HDU accumulation in European countries. This model confirms that favourable temperatures in Europe for HW transmission are mainly found in the southern countries, with an estimated maximum of $10 \mathrm{D}$. Immitis generations per year in the mosquitoes. The model reported represents the first attempt to identify the potential transmission period and the risk areas for HW in Europe.

In case of Theileria parva in Africa, based on climatic suitability for the tick calculated from an interpolated climate database developed for Africa, and "Normalised Difference Vegetation Indices" derived from remotely sensed data, the known distribution of the vector (Rhipicephalus appendiculatus) was compared with the potential distribution, (Perry et al., 1990). The authors concluded that vast areas of Ethiopia, Zaire and the coastal strip of West Africa, from Cameroon to northern Angola, were suitable for the establishment and maintenance of the 
tick, and therefore expresses concern over the role animal movements could play in introducing the vector into new areas.

Bluetongue outbreaks in endemic countries are dependent on the geographical distribution of suitable vectors (Culicoides species) which require specific climatic conditions for their establishment and breeding as well as some of the Culicoides species require cattle dung for breeding. Therefore, if vector distribution maps are available and weather data is recorded for the region under consideration, together with cattle density maps, it should be possible to predict areas where outbreaks are likely to occur (Sanson et al., 1991).

In a case control study of Lyme disease in Baltimore County, Maryland utilizing land use/ land cover maps (derived from Landsat TM imagery) in combination with soils, elevation, geology, and watershed maps to evaluate risk of exposure to Lyme disease and its vector, I. scapularis, it was observed that the risk of disease was significantly lower in highly developed areas, and risk decreased with increasing distance from forests (Glass et al., 1992).

Landsat thematic mapper (TM) data (with 30 m resolution) with geographic information system (GIS) was utilized to identify the high mosquito risk rice fields in California and the canopy of the rice plantation in early season of its growth was correlated with the larval density of Anopheles freeborni. GIS being utilized for measuring the distances between rice fields and source of blood meal for mosquitoes i.e pastures with livestock revealed that the rice fields located near pastures (with livestock) support the production of more larvae than the fields with less developed rice plantations and away from pastures. The accuracy of larval density prediction was possible up to $85 \%$ (Wood et al., 1992).

RS and GIS techniques were also used to identify the landscape features (unmanaged and managed pastures, transitional swamps, mangroves, secondary forests, banana plantations, annual crops, burnt fields, inland water, urban areas and riparian vegetation) up to one $\mathrm{km}$ perimeter (which is usually the flight range of vector mosquito) of 40 villages and correlated it with the density of An. albimanus and malaria incidence. The study used multiband Landsat TM data and colour infrared aerial photography to verify the landscape elements and GIS to create buffer of $1 \mathrm{~km}$ around villages. Stepwise discriminant analysis could identify high risk, low risk and nonmalarious villages with 90\% accuracy (Beck et al., 1994).

Tsetse flies and African trypanosomosis is one such area where satellite data and GIS has been used for risk mapping in smaller regional areas as well as at continental scale (Rogers, 2000). The ability of GIS and RS in capturing spatial temporal data on land use and land cover classes helps to identify the suitability of the land cover classes for tsetse fly habitation.

A study was conducted by Sudhakar et al., (2006) to identify spatial distribution of indicator land cover classes for Phelbotomum argentipes and Kala-azar using RS and GIS techniques in India. Besides this Sangwan et al., (2007) used Rs data to delineate snail habitats in parts of eastern Doon Valley by digital image processing techniques and following FAO framework of land evaluation. They further prepared risk map of snail borne diseases of livestock in Haryana.

Kuerpick et al., (2013) assessed the sero prevalence of F. hepatica in dairy cows in Germany in 2008 for which a total of 20749 bulk tank milk (BTM) samples were collected from all federal states by the dairy factories and state dairy quality control associations for routine checks. Analysis of these samples for antibodies against Fasciola hepatica was done and a geospatial map was drawn for indicating herd prevalence per postal code area by taking into consideration various spatial risk so as to test their potential statistical associations with the ELISA, which resulted in logistic regression supported by a geographical information system (GIS). The GIS indicated that highly positive areas were found in Schleswig-Holstein and the north of Lower Saxony as well as in the southern part of Bavaria. Further, the GIS analysis revealed statistical significant positive associations between the proportion of grassed area and water bodies per postal code area and positive BTM ELISA results. The relation could be attributed to the biology of the amphibious snail Galba (Lymnea) truncatula and the pasture-borne nature of fasciolosis.

\section{Disease mapping}

One among the useful functions of GIS in epidemiology continues to be its disease mapping. When data are collected either routinely or through purposely-designed surveys, and presented in tabular forms, these can then be exploited for analytical usage, but interpretation of such data is often a laborious and time-consuming task and also does not allow easy decision-making (Paolino et al., 2005). Therefore, representation of this data in the form of a map not only facilitates interpretation, but synthesis and recognition of frequency and clusters of phenomena. Disease maps can be drawn to a demographic base which related to the population and the epidemiological information they depict are presented in relation to population size or to a geographic base in which maps are 
constructed according to the shape of a country or a region or any administrative unit. These may be either qualitative in nature e.g. point maps, distribution maps, point distribution maps (PDMs) which indicate location without specifying the amount of disease; or quantitative e.g. distribution maps with proportioned peaks, proportional circle maps, choroplethic maps, choroplethic maps with proportioned peaks, PDMs with proportioned peaks, PDMs with proportioned circles (Rinaldi et al., 2006).

\section{Ecological analysis}

Ecological analysis is targeted on the description of relations existing between the geographic distribution of diseases and environmental risk factors and their analysis by means of statistical procedures (Kistemann et al., 2002). The following fundamental steps could be utilized for making ecological analysis: (i) GIS construction for the study area utilizing data layers on environmental and climatic features; (ii) geo-referencing the geographic units of interest, e.g. farms, centroids of the main pastures, etc.; (iii) creation of buffer zones of a given diameter centered on these geo-referenced points; (iv) extrapolation of values for each environmental feature within each buffer zone; (v) databases with environmental and parasitological data; (vi) statistical analyses (univariate, multivariate, etc.) and individualize of environmental risk factors and/or development of forecast models (Cringoli et al., 2005).

\section{Conclusion}

GIS represent a new technology in veterinary epidemiology for the reporting of animal disease information and the study and modelling of specific disease problems (Sanson et al., 1991). Geographic information systems (GIS) have been used in recent years for a wide variety of purposes like urban and regional planning (Berry \& Berry, 1988) and utility management, land suitability assessment (Diamond \& Wright, 1988), environmental resource monitoring, emergency response management and ecological modelling (Saarenmaa \& Nikula, 1989). Although remote sensing and GIS of bio invasion mapping has lead to the development of a diverse range of mapping technique, but in terms of real world applications, the technology needs further development. Moreover, mapping, modelling and predicting biological invasion is still a major challenge for ecologists due to the involvement of biological processes which are very complex in nature. Though RS helps in formulating hypotheses, but the definitive resolution of problems must be sought at the ground level. The general and definite conclusions based on RS results in the field should not be made without their verification (Daniel et al., 2004).

\section{References}

1) Beck, L.R., Rodriguez, M.H., Dister, S.W., Rodriguez, A.D., Rejmankova, E., Ulloa, A., Meza, R.A., Roberts, D.R., Paris, J.F., Spanner, M.A., Washino, R.K., Hacker, C. and Legters, L.J. 1994. Remote sensing as a landscape epidemiological tool to identify villages at high risk of malaria transmission. American Journal of Tropical Medicine and Hygiene 51: 271.

2) Bedru, S.M. 2006. Remote Sensing and GIS for Land Cover/Land Use Change Detection and Analysis in the Semi-National Ecosystems and Agriculture Landscapes of the Central Ethiopian Rift Valley. Ph.D Dissertation, University of Dresden, Dresden.

3) Berke, O. 2004. Exploratory disease mapping: kriging the spatial risk function from regional count data. International Journal of Health Geographics 26: 18-36.

4) Berry, J.K. and Berry, J.K. 1988. Assessing spatial impacts of land use plans. Journal of Environmental Management 27: 1-9.

5) Biggeri, A., Catelan, D., Dreassi, E., Lagazio, C. and Cringoli, G. 2004. Statistical models for spatial analysis in parasitology. Parassitologia 46: 75-78.

6) Cringoli, G., Rinaldi, L., Veneziano, V. and Musella, V. 2005. Disease mapping and risk assessment in veterinary parasitology: some case studies. Parassitologia 47: 9-25.

7) Daniel, M., Kolar, J. and Zeman, P. 2004. GIS tools for tick and tick-borne disease occurrence. Parasitology 129: S329-S352.

8) Diamond, J.T. and Wright, J.R. 1988. Design of an integrated spatial information system for multiobjective landuse planning. Environment and Planning 15: 205-214.

9) Fortin, J.F. and Slocombe, J.O.D. 1981. Temperature requirements for the development of Dirofilaria immitis in Aedes triseriatus and Aedes vexans. Mosquito News 41: 625-633.

10) Genchi, C., Rinaldi, L., Cascone, C., Mortarino, M. and Cringoli, G. 2005. Is heartworm disease really spreading in Europe? Veterinary Parasitology 133: 137-148. 
11) Glass, G.E., Morgan, J.M., Johnson, D.T., Noy, P.M., Israel, E. and Schwartz, B.S. 1992. Infectious disease epidemiology and GIS: a case study of Lyme disease. Geographical Information Systems 3: 65-69.

12) Guo, H., Huang, Q., Li, X., Sun, Z. and Zhang, Y. 2013. Spatiotemporal analysis of urban environment based on the vegetation-impervious surface-soil model. Journal of Applied Remote Sensing 8: 084597-084597.

13) Janssen, L.L.F. and Bakker, W.H. 2001. Sensors and platforms. In: Principles of Remote Sensing. An Introductory Text Book, pp. 84 - 109 (Janssen, L. L. F. and Huurneman, G. C. eds). International Institute for Aerospace Survey and Earth Sciences. Enschede.

14) Kenneth, E.F. and Magaret, L. 2015. Geographic Information Systems as an Integrating Technology: Context, Concepts, and Definitions. The Geographer's Craft Project, Department of Geography, University of Colorado.

15) Kistemann, T., Dangendorf, F. and Schweikart, J. 2002. New perspectives on the use of Geographical Information Systems (GIS) in environmental health sciences. International Journal of Hygiene and Environmental Health 205: 169-181.

16) Kleinschmidt, I., Bagayoko, M., Clarke, G.P., Craig, M. and Le Sueur, D. 2000. A spatial statistical approach to malaria mapping. International Journal of Epidemiology 29: 355-361.

17) Kuerpick B, Conraths F.J., Staubach C., Fröhlich, A. and Schnieder T. 2013. Seroprevalence and GIS-supported risk factor analysis of Fasciola hepatica infections in dairy herds in Germany. Parasitology 140:1051-60.

18) Kumar, S. 2015. Principles of Remote Sensing. Training for officials of Flood Warning Section, Royal government of Bhutan (20-24 April, 2015).

19) Liu, J.G. and Mason, P.J. 2009. Essential Image Processing for GIS and Remote Sensing. Wiley-Blackwell, a John Wiley \& Sons, Ltd., Publication. United Kingdom. 2009. pp. 443.

20) Lok, J.B. and Knight, D.H. 1998. Laboratory verification of a seasonal heartworm transmission model. In: Seward, R.L. (Ed.), Proceedings of the Heartworm Advances in Heartworm Disease: Symposium'98. American Heartworm Society, Batavia, IL, 15-20.

21) Madelaine, N. 2001. Geographical Information System (GIS) as a Tool in Surveillance and Monitoring of Animal Diseases. Acta Veterinaria Scandinavica 94: 79-85.

22) Maliene, V., Grigonis, V., Palevičius, V. and Griffiths, S. 2011. Geographic information system: Old principles with new capabilities. Urban Design International 16: 1-6.

23) Melesse, A.M., Weng, O., Thenkabail, P.S. and Senay, G.B. 2007. Remote sensing sensors and applications in environmental resources mapping and modelling. Sensors 7: 3209-3241.

24) Menon, S. and Bawa, K. 1997. Application of geographical information systems, remote sensing and a landscape ecology approach to biodiversity conservation in the Western Ghats. Current Science 73: 134141.

25) Nicholson, M.C. and Mather, T.N. 1996. Methods for evaluating Lyme disease risks using geographic information systems and geospatial analysis. Journal of Medical Entomology 33: 711-720.

26) Paolino, L., Sebillo, M. and Cringoli, G. 2005. Geographical information systems and on-line GI Services for health data sharing and management. Parasitologia. 47: 171-175.

27) Perry B.D., Lessard, P., Norval, R.A.I., Kundert, N. and Kruska, R. 1990. Climate, Vegetation and the distribution of Rhipicephalus Appendiculatus in Africa. Parasitology Today 4: 100-104.

28) Pleydell, D.R., Raoul, F., Tourneux, F., Danson, F.M., Graham, A.J., Craig, P.S. and Giraudoux, P. 2004. Modelling the spatial distribution of Echinococcus multilocularis infection in foxes. Acta Tropica 91: 253265.

28) Raso, G., Vounatsou, P., Singer, B.H., N'Goran, E.K., Tanner, M. and Utzinger, J. 2006. An integrated approach for risk profiling and spatial prediction of Schistosoma mansoni-hookworm co-infection. Proceedings of the National Academy of Sciences of USA. 103: 6934-6939.

30) Rinaldi, L., Musella, V., Biggeri, A. and Cringoli, G. 2006. New insights into the application of geographical information systems and remote sensing in Veterinary Parasitology. Geospatial Health 1:33-47.

31) Rogers, D.J. 2000. Satellites, space, time and the African trypanosomiases. Advances in Parasitology 47: 129171.

32) Saarenmaa, H. and Nikula, A. 1989. Managing moose damage in forest plantations: a deep model of animal behaviour on a geographic information system platform. In Proc. 9th International Workshop on Expert Systems and Their Applications, Avignon, May 29-June 2.

33) Sangwan, A.K., Prasad, J. and Jeganathan, C. 2007. Mapping Snail-Borne Parasitic Diseases in a Part of Eastern Doon Valley Using Remote Sensing and GIS Techniques. Indian Congress of Veterinary Parasitology held at Jammu during 7-9 September, India. 
34) Sanson, R.L., Pfeiffer, D.U. and Morris, R. S. 1991. Geographic information systems: their application in animal disease control). Revue scientifique et technique 10: 179-195.

35) Sciarretta, A., Girma, M., Tikubet, G., Belayehun, L., Ballo, S. and Baumgartner, J. 2005. Development of an adaptive tsetse population management scheme for the Luke community, Ethiopia. Journal of Medical Entomology 42:1006-1019.

36) Slocombe, J.O.D., Surgeoner, G.A. and Srivastava, B. 1989. Determination of the heartworm transmission period and its use in diagnosis and control. In: Otto, G.F. (Ed.), Proceedings of the Heartworm Symposium'89. American Heartworm Society, Washington, DC, pp. 19-26.

37) Sudhakar, S., Srinivas, T., Palit, A., Kar, S.K. and Battacharya, S.K. 2006. Mapping of risk prone areas of kalaazar (Visceral leishmaniasis) in parts of Bihar State, India: an RS and GIS approach. Journal of Vector Borne Diseases 43:115-22

38) Ward, M. P. and Carpenter, T. E. 2000. Techniques for analysis of disease clustering in space and in time in veterinary epidemiology. Preventive Veterinary Medicine 45: 257-284.

39) Wood, B.L., Beck, L.R., Washino, R.K., Hibbard, K.A. and Salute, J.S. 1992. Estimating high mosquito producing rice fields using spectral and spatial data. International Journal of Remote Sensing 13: 28132826.

40) Zhang, Z.Y., Xu, D.Z., Zhou, X.N., Zhou, Y. and Liu, S.J. 2005. Remote sensing and spatial statistical analysis to predict the distribution of Oncomelania hupensis in the marshlands of China. Acta Tropica 96: 205-212. 